\title{
Colloidal Assembly on Magnetically Vibrated Stripes
}

\author{
Pietro Tierno, ${ }^{1, *}$ Thomas M. Fischer, ${ }^{2}$ Tom H. Johansen, ${ }^{3}$ and Francesc Sagués ${ }^{1}$ \\ ${ }^{1}$ Departament de Química Física, Universitat de Barcelona, Martí i Franquès 1, 08028 Barcelona, Spain \\ ${ }^{2}$ Institut für Experimentalphysik V, Universität Bayreuth, Bayreuth, Germany \\ ${ }^{3}$ Department of Physics, University of Oslo, P. O. Box 1048, Blindern, Norway
}

(Received 20 December 2007; published 11 April 2008)

\begin{abstract}
We investigate the collective organization of paramagnetic colloidal particles externally driven above the periodic stripes of a uniaxial ferrimagnetic garnet film. An external field modulation induces vibration of the stripe walls and produces random motion of the particles. Defects in the stripe pattern break the symmetry of the potential and favor particle nucleation into large clusters above a critical density. Mismatch between particle size and pattern wavelength generates assemblies with different morphological order. At even higher field strengths, repulsive dipolar interactions between the particles induce cluster melting. We propose a novel approach to generate and externally control a variety of colloidal assemblies.
\end{abstract}

Application of an electric, magnetic, or vibrating drive to a collection of interacting particles in two dimensions (2D) leads to a rich variety of aggregated phases [1,2]. Such studies provide means to understand more general problems in condensed matter like crystal formation, atomic diffusion, or phase transitions. Significant examples include colloidal particles under ac electric fields [3,4], magnetic particles under external modulations $[5,6]$, and vibrating granular media $[7,8]$. While an external homogeneous field imposed on the particle ensemble elicits global responses, spatially periodic potentials can act individually on the particles allowing a more precise control over their mutual interactions. Optical tweezers [9] or structured magnetic substrates [10] can be used to generate heterogeneous potentials on the colloidal length scale. In the latter case, a ferrimagnetic garnet film with stripe domains provides a 1D periodic pinning landscape on which one can trap [10] or move [11] arrays of paramagnetic particles. In the last case, the interplay between magnetic interactions and viscous damping is the crucial factor of the particle behavior. By further reducing the spatial length scale of the confining potential, we here demonstrate that a time periodic magnetic driving force leads to an ordered aggregation of colloidal particles. This aggregation thermally relaxes after turning off the applied field. Moreover, controlled reversibility (assembly or disassembly) is gained by externally varying the amplitude of the imposed magnetic modulation.

We study the induced aggregation of paramagnetic colloids deposited on a garnet film having alternating ferromagnetic domains with wavelength smaller and incommensurate with respect to the particle size. Externally induced vibration of the domain walls (DWs) causes random particle motion on the surface. By increasing the particle concentration, we observe cluster formation around regions where high stripe curvature or point defects break the symmetry of the periodic potential. By varying the intensity and frequency of the external mag- netic field, we observe a variety of morphological assemblies and report on the system full phase diagram. At higher field strengths, cluster melting occurs due to repulsive dipolar forces.

In our experiments, we used a bismuth substituted ferrite garnet film with uniaxial magnetic anisotropy which was grown by liquid phase epitaxy. At equilibrium, the film is structured in ferromagnetic domains having alternating magnetization normal to the film, with a wavelength $\lambda=$ $1.7 \pm 0.1 \mu \mathrm{m}$, twice the stripe width, Fig. 1(a). The magnetic field between neighboring domains is directed above the film from domains with up magnetization towards domains with down magnetization. Micro cracks are present in the film in form of straight cuts which are created during the growth process [12], and divide the film into

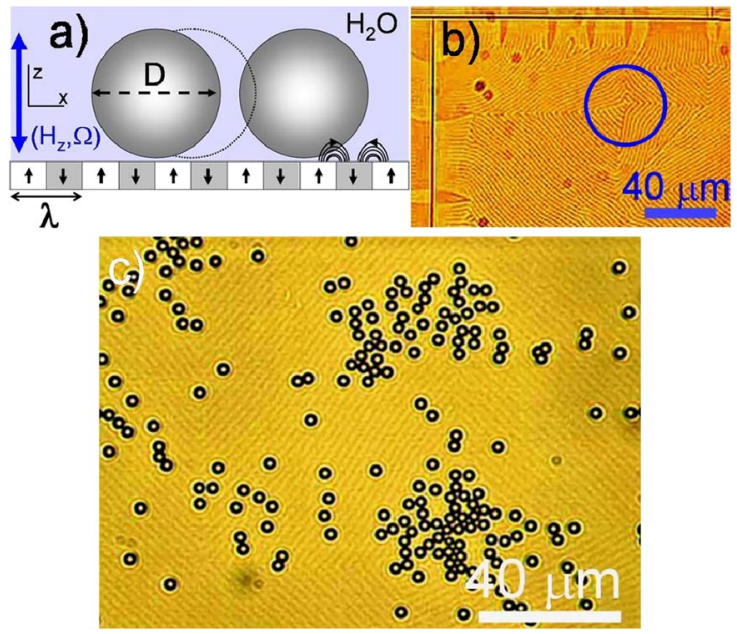

FIG. 1 (color online). (a) Schematic picture of two paramagnetic particles (diameter $D$ ) on a magnetic stripe pattern of wavelength $(\lambda=0.62 D)$ under a magnetic field $\mathbf{H}_{\mathbf{z}}$ oscillating with frequency $\Omega$ along $z$. (b) Image of the garnet film showing crack lines and a stripe defect. (c) Polarization microscope image of the particles on the film. 
several areas $\left(\sim 5 \times 10^{4} \mu \mathrm{m}^{2}\right)$. The domains in the garnet can pack into large regions where straight parallel stripes of different orientations merge by bending [see small circle in Fig. 1(b)]. Application of an oscillating magnetic field $\mathbf{H}_{\mathrm{ext}}=H_{z} \sin (\Omega t) \mathbf{e}_{z}$ normal to the film, with frequency $\Omega$ and amplitude $H_{z}$, produces a periodic widening (shrinking) of domains with parallel (antiparallel) magnetizations, and therefore results in a planar vibration of the DWs [13] (Video 1 in [14]). This vibration, analyzed in terms of the amplitude displacement $\Delta x$ of one such DW, depends linearly $\left(\Delta x / H_{z} \sim 3 \times 10^{-10} \mathrm{~m}^{2} / \mathrm{A}\right)$ on the field amplitude (Fig. 1 in [14]). Thus, we used frequency and amplitude of the external field to control the particle clustering.

Colloids used were polystyrene paramagnetic particles with a diameter $D=2.8 \mu \mathrm{m}$ and effective magnetic susceptibility $\chi=0.17$ (Dynabeads M-270). A water drop containing a large number of particles (from $10^{7}$ to $5 \times$ $10^{8}$ beads $/ \mathrm{ml}$ ) was placed on the garnet film. Once deposited, the colloidal particles tend to align along the DWs, where the stray field is maximal, although the excluded volume $(\lambda$ is $0.62 D$ ), forbids the formation of parallel chains along contiguous DWs, Fig. 1(c). The particles are also confined by the crack lines inside the square areas, and this allows us to define an areal density, $\rho=N / A, N$ being the number of particles contained in area $A$. The external magnetic modulation was provided by a coil placed under the garnet film. Video-microscopy and particle tracking routines were used to follow the individual particle trajectories and to statistically analyze the collective system evolution.

Application of the external magnetic field continuously shakes the particles which, as a result, perform a 1D random walk normal to the stripes, (Video 2 in [14]). In an ideal situation with infinite parallel stripes, the particles would continue to move without any chance to aggregate. In the film, stripes curve and form magnetic singularities of the stray field which attract the colloidal particles. Stripe curvature [15] introduces a bias into the wandering motion of the colloidal particles which prefer to collect at the defect location instead of randomly disperse above the film (in Fig. 2 in [14], we show the mean square displacements of particles close to and far from a defect). This is a typical feature of heterogeneous nucleation favored by substrate defects. In Fig. 2, we show two images of an assembly of particles with $\rho=0.048 \mu \mathrm{m}^{-2}$, right at the time of application of an external field (a) and $6.5 \mathrm{~s}$ later (b) (Video 3 in [14]).

To characterize the cluster growth, we tracked the particle positions at each frame and determined the number fraction $\phi_{N}=N_{p} / N$ with $N_{p}$ the number of particles inside a cluster. $N_{p}$ was calculated by evaluating the paircorrelation function $g(r)$ at each frame and using as clustering condition a criterion [16] based on the position of the first peak of $g(r)$. Figure 2(c) shows how $\phi_{N}$ varies with time for three field frequencies. Aggregates reach their final size $\left(\phi_{N}=\right.$ const $\left.\equiv \phi_{N}^{A}\right)$ faster when increasing the
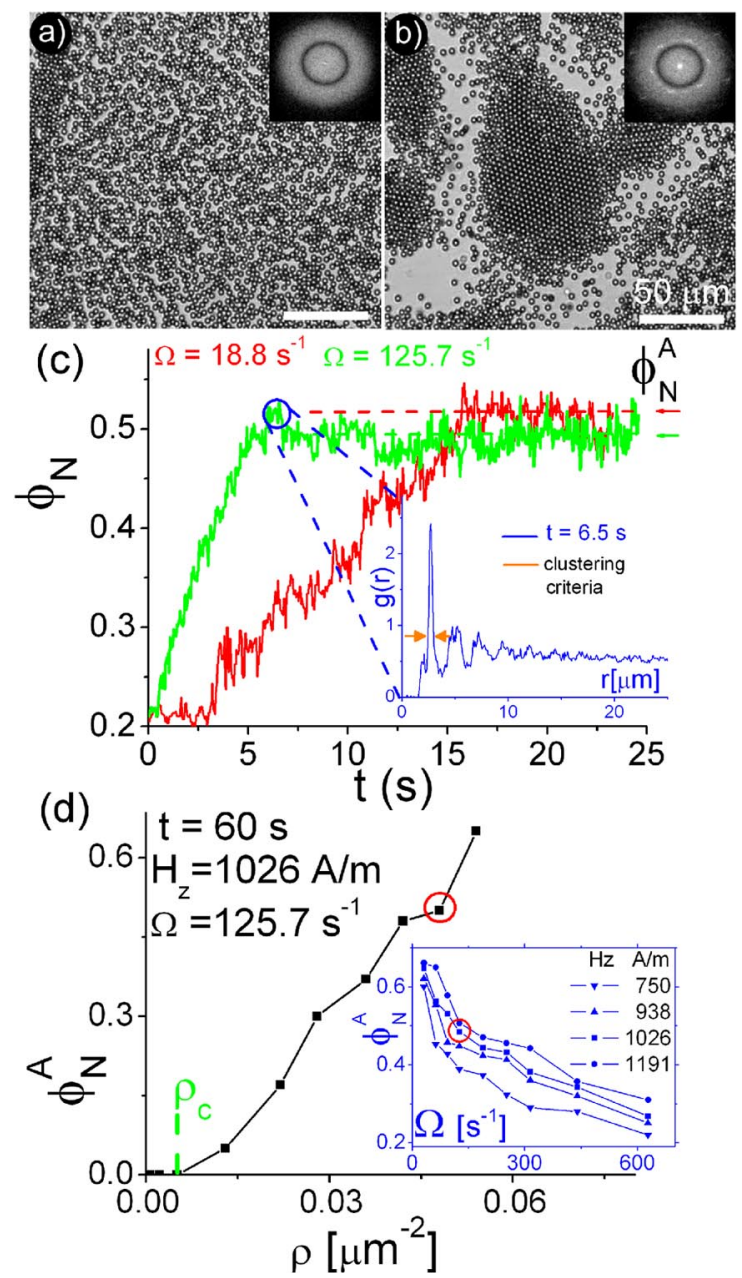

FIG. 2 (color online). Microscope images of an assembly of particles with $\rho=0.048 \mu \mathrm{m}^{-2}$ under a field $H_{z}=1026 \mathrm{~A} / \mathrm{m}$ $\left(\Omega=125.7 \mathrm{~s}^{-1}\right)$ at $t=0 \mathrm{~s} \mathrm{(a)}$ and $t=6.5 \mathrm{~s}(\mathrm{~b})$. Small insets on top of the images correspond to FFT spectra. (c) Number fraction $\phi_{N}$ versus time $t$ at frequencies $\Omega=18.8 \mathrm{~s}^{-1}, 125.7 \mathrm{~s}^{-1}$. The small inset shows $g(r)$ at $6.5 \mathrm{~s}$ corresponding to the image in (b). (d) $\phi_{N}^{A}$ versus areal density. The inset shows $\phi_{N}^{A}$ versus driving frequency for different magnetic field strengths $(\rho=$ $0.048 \mu \mathrm{m}^{-2}$ ).

frequency, and after $\sim 20 \mathrm{~s}$, most of the final clusters in our experiments were formed. We could confirm the presence of pattern defects underlaying the cluster centers when switching off the field which allows the particles to slowly escape along the DWs by thermal diffusion. We did not observe coarsening, a signature that strong pinning conditions imposed by the underlying defects impeded depletion of small clusters in favor of larger ones. In Fig. 2(d), we explore the role of density in cluster formation at constant strength and frequency of the external field. As expected, larger areal densities increase $\phi_{N}^{A}$. Below a critical value $\rho_{c}$, the driven particles move on the film without aggregating, although inelastic collisions between the particles occurs during motion. Presence of a threshold density for cluster formation is similar to granular materials under driving forces [17]. We observe a slight continu- 
ous increase of $\phi_{N}^{A}$ with the imposed magnetic intensity but a decrease with increasing frequency being substantial at high frequencies [ $\Omega>150 \mathrm{~s}^{-1}$, see inset in Fig. 2(d)], the latter feature being understood as a limitation of the damped particles to follow too fast modulations.

An important feature of our system relies in the incommensurate ratio $\lambda / D$. In the case of substrates with static pinning lines, mismatch has been advantageously used to grow different types of colloidal crystals $[18,19]$. In the present work, the pinning lines dynamically vibrate, and different colloidal structures were observed by varying the strength and frequency of the imposed field. More specifically, we observed the formation of hexagonal, square, chainlike, and network type assemblies. The phase diagram $\left(\Omega, H_{z}\right)$ encompassing all these structures for a density $\rho=0.032 \mu \mathrm{m}^{-2}$ and after $\sim 60 \mathrm{~s}$ forcing, is shown in Fig. 3. For field intensities lower than $\sim 400 \mathrm{~A} / \mathrm{m}$, small stripe displacements are unable to provide sufficient kinetic energy to the particles, and no specific pattern was observed. For fields up to $\sim 1500 \mathrm{~A} / \mathrm{m}$, nucleation occurs with predominantly square $\left(\Omega<20 \mathrm{~s}^{-1}\right)$ or hexagonal $\left(20 \mathrm{~s}^{-1}<\Omega<150 \mathrm{~s}^{-1}\right)$ order (note a small island of square order at $40 \mathrm{~s}^{-1}<\Omega<$ $60 \mathrm{~s}^{-1}$ ). We sometimes observed some hysteresis or coexistence of both phases. To distinguish between them, we used a criterion formulated in Ref. [20]. We perform a Voronoi-like analysis for each particle position and measure the average area $\langle a\rangle$ and perimeter $\left\langle\prod\right\rangle$ of the Voronoi cells for all particles in the cluster. We further employ the circularity index as $\kappa=2 \sqrt{\pi} \sqrt{\langle a\rangle} /\left\langle\prod\right\rangle$, with $\kappa \sim 0.95$ $(\kappa \sim 0.89)$ for ideal hexagonal (square) packing. In our case, we classify order as hexagonal when $\kappa>0.92$ and otherwise for square. At low frequencies, most of the particles try to follow the oscillating DWs, and the resulting square patterns are built from the frustrated motion normal to the DWs inside the cluster. Increasing further the field enhances DW displacements destroying the regular cluster arrangement. The moving particles in this case form a connected network whose mesh size is comparable to $\Delta x_{M}$. For fast modulations, particles cannot follow the stripe vibrations, and what they feel is just an average attraction towards the local energy minima located on the pattern defects. Hence, the configuration adopted is the most symmetric, hexagonal-like. At higher fields, particles assemble into repelling chains disposed parallel to each other. The damped particles dispose along the average position of the fast vibrating DWs which, at these field strengths, present larger separation. The chains are metastable, and particles can exchange position between consecutive lines giving rise to oscillations of the chains along their long axis. The interchain distance, which is $\sim 4 \mu \mathrm{m}$ for Fig. 3(d), depends on particle concentration.

In contrast to colloidal crystals nucleated by previously cited techniques, we find that cluster assembly is reversible in our situation, and cluster melting is obtained when $H_{z}$ exceeds a critical value $H_{c}$.
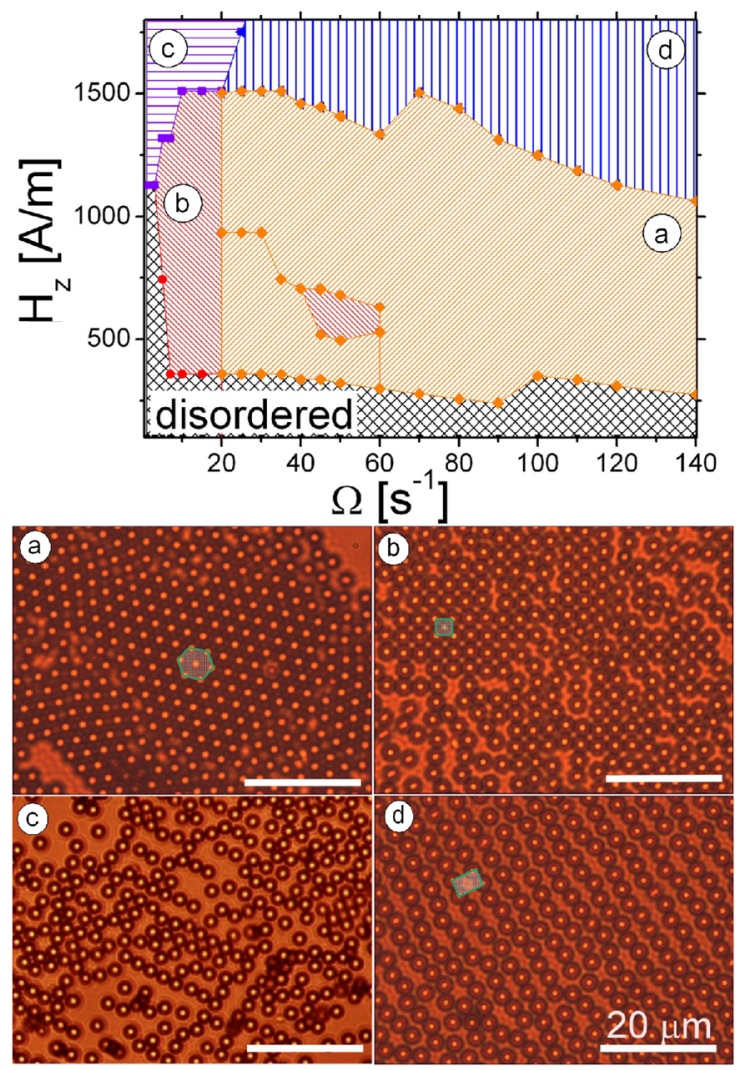

FIG. 3 (color online). Phase diagram in the $\left(\Omega, H_{z}\right)$ plane for an areal density $\rho=0.032 \mu \mathrm{m}^{-2}$ and after $t \sim 60 \mathrm{~s}$. The various regions show colloidal assemblies with order: (a) hexagonal, (b) square, (c) network, (d) chainlike. On the bottom, microscope images of the different modes of assembly.

In Fig. 4, we show microscope images of a hexagonal cluster subjected to a field of $1.3 \times 10^{4} \mathrm{~A} / \mathrm{m}$ and a frequency of $\Omega=125.7 \mathrm{~s}^{-1}$ initially (a) and $5.7 \mathrm{~s}$ later (b) (Video 4 in [14]). Under such strengths, particles escape from the cluster perimeter and distribute evenly over the film. Stripes also disappear, and the film is magnetically saturated by the external field. To estimate the critical field $H_{c}$ for this transition, we measure the position of the first peak $r_{p}$ of $g(r)$, and plot it as a function of the applied field $H_{z}$ [see Fig. 4(c)]. In the small inset on top of the graph, we show $g(r)$ for two field strengths, below and above $H_{c}$ around which $r_{p}$ changes from $2.8 \mu \mathrm{m}(\sim D)$ to $3.1 \mu \mathrm{m}$ [21].

The aggregation and melting process admit an interpretation based on the interparticle interactions. A paramagnetic particle of volume $V$ subjected to a field $\mathbf{H}$ acquires a magnetic moment $\mathbf{m}=V \chi \mathbf{H}$ and a magnetic energy $E=$ $-\mu \mathbf{m} \cdot \mathbf{H}$, where $\mu$ is the water magnetic susceptibility. The pair-potential between two dipoles $\mathbf{m}_{i}, \mathbf{m}_{j}$ separated by $\mathbf{r}_{i j}$ reads, $U_{d d}=\frac{\mu}{4 \pi}\left(\frac{\mathbf{m}_{i} \cdot \mathbf{m}_{j}}{\mathbf{r}_{i j}^{3}}-\frac{3\left(\mathbf{m}_{i} \cdot \mathbf{r}_{i j}\right)\left(\mathbf{m}_{j} \cdot \mathbf{r}_{i j}\right)}{\mathbf{r}_{i j}^{5}}\right)$. Such interaction is maximally attractive (repulsive) for particles with moment parallel (normal) to $\mathbf{r}_{i j}$. Here, the total field which dictates the dipole directions results from $\mathbf{H}_{\text {ext }}$ (along $z$ ) 


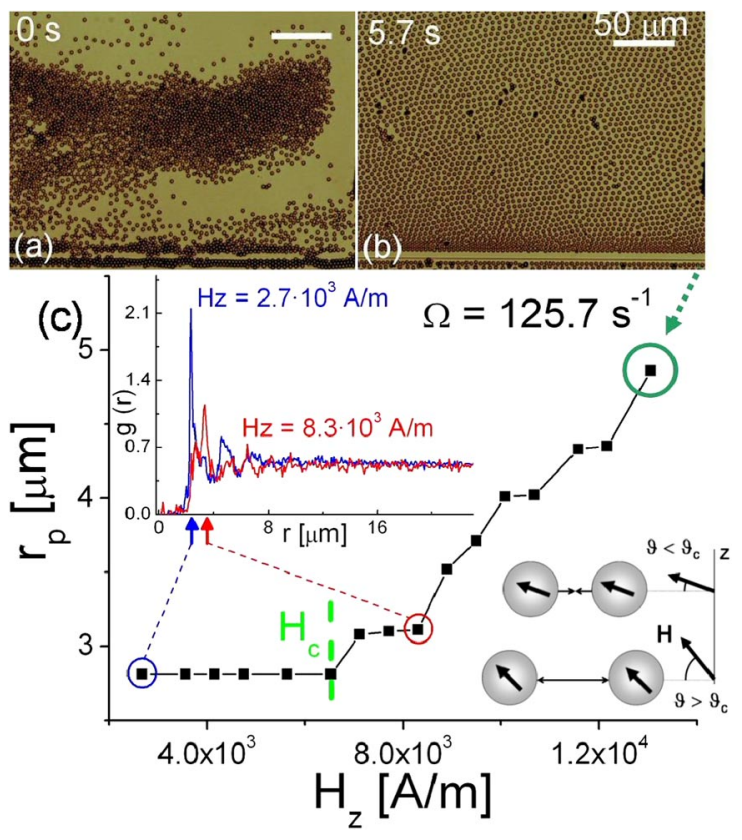

FIG. 4 (color online). Microscope images of a preexisting cluster subjected to $H_{z}=1.3 \times 10^{4} \mathrm{~A} / \mathrm{m}$ at $t=0 \mathrm{~s}$ (a) and $t=$ $5.7 \mathrm{~s}$ after forcing (b). (c) First peak position $r_{p}$ of asymptotic $g(r)$ (top inset) versus $H_{z}$. All clusters were obtained by successive assembly or disassembly of the aggregate in (a). Bottom inset shows a pictorial of dipole interactions.

and the garnet contribution at the DW (in the plane $x, y$ ), and thus becomes tilted. In fact, similar patterns in form of distorted square or chainlike assemblies have been previously observed [22] and computed [23] for dipolar magnetic particles under a static tilted magnetic field. To be energetically favorable, the induced moments should assume a configuration of antisymmetric chains (Fig. 3 in [14]) due to the underlying direction of the stray fields. If we define $\vartheta$ as the tilt angle for the dipole orientations relative to the particle plane $(x, y)$, one can estimate, $U_{\mathrm{dd}} \propto$ $\left(6-11 \cos ^{2} \vartheta\right)$, for nearest-neighbor interactions in a unit cell of a hexagonal lattice. Depending on $H_{z}, \vartheta$ can be lower or higher than the critical angle at which dipolar interactions turn from attractive to repulsive, $\vartheta_{c} \sim 43^{\circ}$ [inset Fig. 4(c)]. From the evaluated critical field $H_{c}$ for cluster melting, one can estimate the average contribution of the garnet film to the total magnetic field to be $\sim 4300 \mathrm{~A} / \mathrm{m}$.

In conclusion, we have investigated the collective assembly of paramagnetic colloidal particles deposited on a stripe potential subjected to the oscillation of an external magnetic field. The proposed method provides a model system to study phenomena of 2D heterogeneous nucleation driven by substrate defects with direct observation and controlled on a single particle level. In this way, our work expands to 2D recent experimental [24] and theoretical [25] investigations of heterogeneous nucleation in 3D col- loidal bulk suspension. Also, the observed assemblies will allow to study phase transitions between different morphological orders or to easily build two-dimensional regular colloidal patterns with variable interparticle distances, which could be of interest in photonic band gap applications.

The authors acknowledge J. I. Mullol for experimental support. P. T. was supported by the program 'Beatriu de Pinós No. BP-B100167. F. S. and P. T. acknowledge financial support by MEC (Project No. FIS2006-03525) and DURSI No. (2005SGR00653), T.M.F. by the National Science Foundation under No. CHE-0649427, and T. H. J. by The Research Council of Norway.

*ptierno@ub.edu

[1] M. Trau, D. A. Saville, and I. A. Aksay, Science 272, 706 (1996).

[2] I. S. Aranson and L. S. Tsimring, Rev. Mod. Phys. 78, 641 (2006).

[3] S.-R. Yeh, M. Seul, and B. I. Shraiman, Nature (London) 386, 57 (1997).

[4] K.-Q. Zhang and X. Y. Liu, Nature (London) 429, 739 (2004).

[5] G. Helgesen et al., Phys. Rev. Lett. 61, 1736 (1988).

[6] P. Tierno, R. Muruganathan, and T. M. Fischer, Phys. Rev. Lett. 98, 028301 (2007).

[7] J. S. Olafsen and J. S. Urbach, Phys. Rev. Lett. 81, 4369 (1998).

[8] J. Li et al., Phys. Rev. Lett. 90, 134301 (2003).

[9] K. Mangold, P. Leiderer, and C. Bechinger, Phys. Rev. Lett. 90, 158302 (2003).

[10] L. E. Helseth et al., Langmuir 21, 7518 (2005).

[11] P. Tierno et al., Phys. Rev. E 75, 041404 (2007).

[12] L. E. Helseth et al., Appl. Phys. Lett. 85, 2556 (2004).

[13] J.C. Slonczewski, B.E. Argyle, and J. H. Spreen, IEEE Trans. Magn. 17, 2760 (1981).

[14] See EPAPS Document No. E-PRLTAO-100-087815 for supplementary videos. For more information on EPAPS, see http://www.aip.org/pubservs/epaps.html.

[15] P. Dhar et al., J. Phys. Chem. B 111, 13097 (2007).

[16] E. K. Hobbie, Phys. Rev. Lett. 81, 3996 (1998).

[17] A. Kudrolli, M. Wolpert, and J. P. Gollub, Phys. Rev. Lett. 78, 1383 (1997).

[18] K.-H. Lin et al., Phys. Rev. Lett. 85, 1770 (2000).

[19] M. Allard et al., Adv. Mater. 16, 1360 (2004).

[20] C. Gollwitzer, I. Rehberg, and R. Richter, J. Phys. Condens. Matter 18, S2643 (2006).

[21] A future study will investigate whether $H_{c}$ depends on the forcing frequency or initial morphology.

[22] W. Wen, L. Zhang, and P. Sheng, Phys. Rev. Lett. 85, 5464 (2000).

[23] V. A. Froltsov et al., Phys. Rev. E 68, 061406 (2003).

[24] V. W. A. de Villeneuve et al., Science 309, 1231 (2005).

[25] A. Cacciuto, S. Auer, and D. Frenkel, Nature (London) 428, 404 (2004). 